\title{
Corrigendum
}

Tim V.P. Bliss, Graham L. Collingridge, Richard G.M. Morris and Klaus G. Reymann

\section{Corrigendum to: Long-term potentiation in the hippocampus: discovery, mechanisms and function}

https://doi.org/10.1515/nf-2018-2002

Corrigendum to: Tim V.P. Bliss, Graham L. Collingridge, Richard G. M. Morris and Klaus G. Reymann. 2018. Longterm potentiation in the hippocampus: discovery, mechanisms and function. Neuroforum. Volume 24, Issue 3, pages A103-A120. (DOI: https://doi.org/10.1515/nf-2017-A059)
The following historically important reference is missing in the reference list of the original articles:

Bliss, T.V.P. and Gardner-Medwin, A.R. (1973). Longlasting potentiation of synaptic transmission in the dentate area of the unanaesthetized rabbit following stimulation of the perforant path. Journal of Physio$\log y 232,357-374$.

*Corresponding authors: Tim V.P. Bliss, The Frances Crick Institute, London, UK, tim.bliss@crick.ac.uk

*Graham L. Collingridge, Department of Physiology, University of Toronto, Canada, Lunenfeld-Tanenbaum Research Institute, Mount Sinai Hospital, Toronto, Canada, Centre for Synaptic Plasticity, University of Bristol, UK, glcollingridge@gmail.com

*Richard G.M. Morris, Centre for Discovery Brain Sciences, Edinburgh Neuroscience, University of Edinburgh, UK, R.G.M.Morris@ed.ac.uk *Klaus G. Reymann, Leibniz Institute for Neurobiology and Center for Behavioral Brain Sciences, Neuropharmacology, Magdeburg, Germany, klaus.reymann@t-online.de 